\title{
熵工程在热电材料中的应用
}

\author{
杨青雨 ${ }^{1,2}$, 仇鹏飞 ${ }^{1,2}$, 史 迅 ${ }^{1,2}$, 陈立东 ${ }^{1,2}$ \\ (1. 中国科学院 上海硅酸盐研究所, 上海 200050; 2. 中国科学院大学 材料科学与光电技术学院, 北京 100049)
}

摘 要: 作为高摘合金设计思想的延伸, 摘工程可从电子和声子输运两方面引导热电材料的性能优化, 在多种热电 材料体系已经获得了成功应用。特别是, 熵具有内禀的类似基因特性, 可以作为热电材料的指征因子, 对多元热电 材料实现快速篮选。本文首先揭示熵作为热电材料基因特性的内禀原因, 阐述构型摘增加导致材料晶体结构对称性 增强、泽贝克系数提升、晶格热导率下降的物理机制; 然后着重介绍摘工程在类液态材料和 IV-VI 族半导体等典型 热电材料体系中的应用，总结熵工程提高材料热电性能的研究进展; 并介绍多元单相高熵热电材料的热力学稳定 性预测方法; 最后指出了熵工程将来的研究重点。

关 键 词: 熵工程; 热电材料; 材料基因工程; 电声输运; 综述

中图分类号: TB34 文献标识码: A

\section{Application of Entropy Engineering in Thermoelectrics}

\author{
YANG Qingyu $^{1,2}$, QIU Pengfei ${ }^{1,2}$, SHI Xun ${ }^{1,2}$, CHEN Lidong ${ }^{1,2}$
}

(1. Shanghai Institute of Ceramics, Chinese Academy of Sciences, Shanghai 200050, China; 2. Center of Materials Science and Optoelectronics Engineering, University of Chinese Academy of Sciences, Beijing 100049, China)

\begin{abstract}
As the extension of high-entropy alloy, entropy engineering has been already extensively used in thermoelectrics because it can guide the optimization of thermoelectric (TE) performance from the aspects of both electrical and thermal transports. Due to the inherent material gene-like feature, entropy can be used as a performance indicator to rapidly screen new multicomponent TE materials. In this review, we first reveal the reason why entropy can be used as the performance indicator of TE materials. The physical mechanisms of enhanced structure symmetry, improved Seebeck coefficient, and suppressed lattice thermal conductivity as a result of the increased configurational entropy are discussed. Then, the applications of entropy engineering in typical TE materials, such as liquid-like materials and IV-VI semiconductors, are outlined, and the approach to screen and identify candidate multicomponent TE materials with high configurational entropy is introduced. Finally, the future directions for entropy engineering are highlighted.
\end{abstract}

Key words: entropy engineering; thermoelectric material; materials genome engineering; electrical and phonon transports; review

热电技术是一种基于泽贝克效应和帕尔贴效应 实现电能和热能直接相互转换的新型清洁能源技术,
具有无移动组件、无噪音、高可靠性等优点, 在汽 车尾气和工业余废热的回收利用、深空探测设备的

收稿日期: 2020-07-27; 收到修改稿日期：2020-09-14; 网络出版日期：2020-10-19

基金项目: 国家杰出青年科学基金(51625205)

National Science Foundation for Distinguished Young Scholars (51625205)

作者简介: 杨青雨(1995-), 男, 博士研究生. E-mail: yangqingyu@student.sic.ac.cn

YANG Qingyu(1995-), male, PhD candidate. E-mail: yangqingyu@student.sic.ac.cn

通信作者：史 迅，研究员. E-mail: xshi@mail.sic.ac.cn

SHI Xun, professor. E-mail: xshi@mail.sic.ac.cn 
电源供给、通讯设备的微型芯片制冷等领域具有广 阔的应用前景 ${ }^{[1]}$ 。热电技术能量转换效率主要由无量 纲热电优值决定, 其定义为: $Z T=S^{2} \sigma T /\left(\kappa_{\mathrm{e}}+\kappa_{\mathrm{L}}\right)$, 其中 $S$ 为泽贝克系数, $\sigma$ 为电导率, $T$ 为热力学温度, $\kappa_{\mathrm{e}}$ 为载流子热导率, $\kappa_{\mathrm{L}}$ 为晶格热导率。高的转换效 率一般要求高泽贝克系数和高电导率, 以及低晶格 热导率, 但是, 这三个参数相互耦合, 对热电材料 $Z T$ 值的提升极具挑战。例如, 增加泽贝克系数一般会 伴随电导率的降低, 而降低晶格热导率又会对载流 子造成散射, 降低电导率。因此, 发现电热输运解耦 新机制, 实现电热输运的协同调控是热电性能优化 的核心目标和难题 ${ }^{[2]}$ 。

最近二十年, 研究人员陆续提出诸如 “电子晶 体-声子玻璃 ${ }^{[3]}$ ”、纳米工程 ${ }^{[4]}$ 、声子共振散射 ${ }^{[5-7]}$ 、 能带工程 ${ }^{[8]}$ 、类液态效应 ${ }^{[9]}$ 、熵工程 ${ }^{[10]}$ 等电热输运 解耦新机制, 将热电材料的 $Z T$ 值从 1.0 逐步提高到 2.0 左右。其中, 熵工程源于高熵合金的设计思想 ${ }^{[11]}$ 。 高熵合金是指由五种或五种以上等量或大约等量金 属形成的合金 ${ }^{[12-13]}$ ，具有四大效应：热力学上的高 熵效应, 动力学上的迟滞扩散效应, 结构上的晶格 畸变效应, 以及性能上的“鸡尾酒”效应 ${ }^{[14]}$ 。这些效 应使高熵合金具有超越传统合金的优良力学性能, 从而获得了研究人员的高度关注 ${ }^{[15]}$ 。近十年来, 研 究发现高熵合金的四大效应不仅可以优化金属材料 的力学性能, 而且可以改善无机非金属材料的多项 物理性能。因此, 摘工程的设计思路从金属材料领 域逐渐延伸至软磁 ${ }^{[16]}$ 、热磁 ${ }^{[17]}$ 、超导 ${ }^{[18]}$ 、储氢 ${ }^{[19]}$ 、 介电材料 ${ }^{[20]}$ 、锂电池 ${ }^{[21]}$ 、热电 ${ }^{[10,22]}$ 等无机非金属功 能材料领域。2015 年, Shafeie 等 ${ }^{[22]}$ 研究了 $\mathrm{Al}_{x} \mathrm{CoCrFeNi}$ 高熵合金的热电性能。2017 年, $\mathrm{Liu}$ 等 ${ }^{[10]}$ 深入阐述了摘与热电材料的电热输运参数之间的关 系, 提出熵可以作为热电材料的“基因”参量, 进而 对多元热电材料实现快速篮选和设计。如图 1 所示,

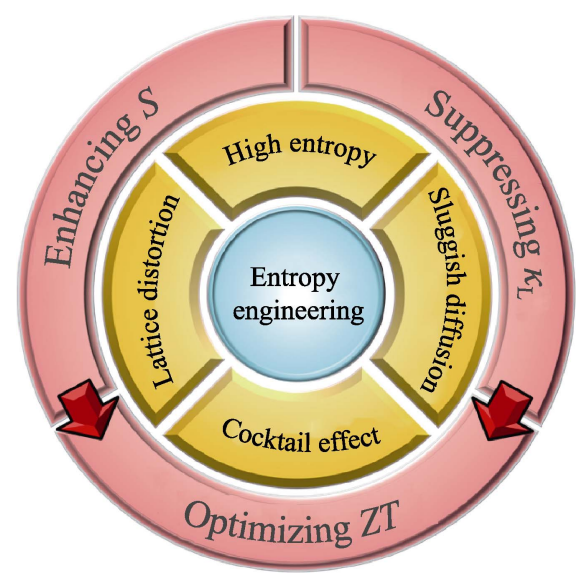

图 1 熵工程在热电材料中的应用示意图

Fig. 1 Schematic of entropy engineering in thermoelectrics
通过对熵的调控，可以从电子和声子的输运等多方 面指导热电材料的性能优化。此后, 熵工程的设计 理念被应用于类液态热电材料、IV-VI 族化合物等 多种热电材料体系, 并成功提升热电性能。本文首 先揭示熵作为热电材料基因特性的内禀原因, 然后 以几个典型材料体系为例总结熵工程提高材料热电 性能的研究进展, 最后介绍如何在理论上预测稳定 的新型单相高熵热电材料。

\section{1 熵与电热输运性质的关联}

\section{1 熵与电输运性质的关联}

如图 2 所示, 增加构型熵可以提高材料晶体结 构对称性, 进而提高泽贝克系数。根据玻尔兹曼理 论，构型熵可表示为 ${ }^{[23]}$.

$$
\Delta S=k_{\mathrm{B}} \ln \Omega=-N_{\mathrm{A}} k_{\mathrm{B}} \sum_{i=1}^{n} x_{i} \ln x_{i}
$$

其中, $k_{\mathrm{B}}$ 为玻尔兹曼常数, $\Omega$ 为可能的微观态数, $x_{i}$ 是第 $i$ 个组分的摩尔含量, $N_{\mathrm{A}}$ 为阿伏伽德罗常 数。由公式(1)可知, 当增加材料中元素组成时, 其 构型熵增加。构型熵高会使晶格内原子分布更加无 序并在宏观尺度上更均匀, 从而材料更易于形成高 对称性晶体结构。具有高对称性晶体结构的材料在 实空间和倒空间中都存在许多等效位点, 能够形成 高简并的带边以及与对称性相关的多能谷电子口袋， 有助于提高材料的有效质量和泽贝克系数, 进而获 得更好的电输运性能 ${ }^{[10]}$ 。

Liu 等 ${ }^{[10]}$ 从理论上揭示了构型熵增加导致材料 高对称性晶体结构向低温移动的原因。以二元固溶 体系为例, 当 $\mathrm{AB}$ 和 $\mathrm{A}^{\prime} \mathrm{B}$ 两种材料形成 $\mathrm{A}_{1-\chi} \mathrm{A}^{\prime}{ }_{x} \mathrm{~B}$ 固 溶体时, 即 $(1-x) \mathrm{AB}+x \mathrm{~A}^{\prime} \mathrm{B} \rightarrow \mathrm{A}_{1-x} \mathrm{~A}_{x}{ }_{x} \mathrm{~B}$, 一个 $\mathrm{A}^{\prime} \mathrm{B}$ 溶 质晶胞取代 $\mathrm{AB}$ 基体晶胞将产生额外的焓变 $\Delta H_{\text {cell }}$ 。

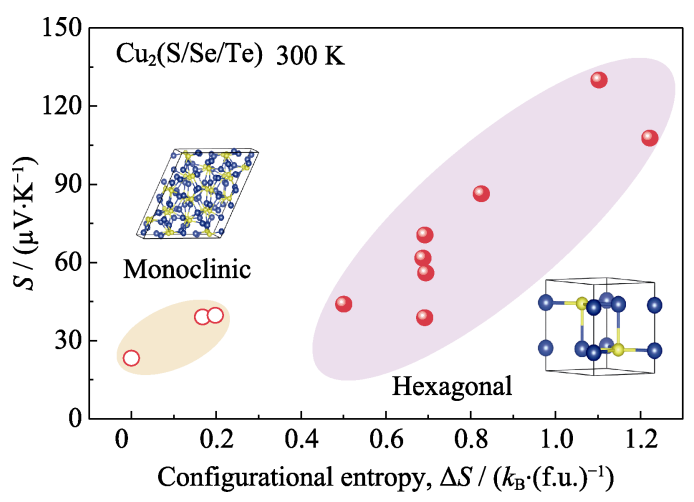

图 $2 \mathrm{Cu}_{2}(\mathrm{~S} / \mathrm{Se} / \mathrm{Te})$ 体系泽贝克系数与构型熵的关系 ${ }^{[10]}$

Fig. 2 Room-temperature Seebeck coefficient as a function of configurational entropy in $\mathrm{Cu}_{2}(\mathrm{~S} / \mathrm{Se} / \mathrm{Te})$-based multicomponent materials $^{[10]}$ 
$\Delta H_{\text {cell }}$ 由原子电荷转移造成的电荷转移能 $\Delta H_{\text {cell }}^{\mathrm{c}}$ 和 原子位置偏移引起的晶格畸变能 $\Delta H_{\mathrm{cell}}^{\mathrm{s}}$ 两部分组 成。在等电子固溶过程中, 电荷转移能 $\Delta H_{\mathrm{cell}}^{\mathrm{c}}$ 远小于 晶格畸变能 $\Delta H_{\text {cell }}^{\mathrm{s}}$ 。因此, 在固溶焓变的计算中, 电 荷转移能的贡献可以忽略。基于高摘合金的连续体 模型和弹性力学模型, 晶格畸变能 $\left(\Delta H_{\mathrm{s}}\right)$ 表示为 ${ }^{[24-25]}$ :

$$
\Delta H_{\mathrm{s}}(x)=x \int_{1}^{x} \frac{\Delta H_{\text {cell }}^{\mathrm{s}}(t)}{Z} \cdot N_{\mathrm{A}} \mathrm{d} t+(1-x) \int_{0}^{x} \frac{\Delta H_{\text {cell }}^{\mathrm{s}}(t)}{Z} \cdot N_{\mathrm{A}} \mathrm{d} t
$$

式中, $\Delta H_{\text {cell }}^{\mathrm{s}}$ 为 $\mathrm{A}^{\prime} \mathrm{B}$ 溶质晶胞替换 $\mathrm{AB}$ 基体晶胞所需 能量。当 $x=0.5$ 时, $\Delta H_{\mathrm{s}}$ 取得最大值, 其二阶泰勒展 开式为 ${ }^{[10,24-25]}$.

$$
\begin{aligned}
& \Delta H_{\mathrm{s}}(x) \approx M N_{\mathrm{A}} \cdot\left[\bar{G} \bar{R}^{*}\left(\Delta R^{*}\right)^{2} / \mathrm{Z}\right] \cdot(1-x) x= \\
& \quad M N_{\mathrm{A}} \cdot \delta \cdot(1-x) x
\end{aligned}
$$

其中, $\delta$ 为溶解度因子 $\left(\delta=\bar{G} \bar{R}^{*}\left(\Delta R^{*}\right)^{2} / Z\right) ; R^{*}$ 为 有效晶格常数; $\bar{G}$ 和 $\bar{R}$ 分别为 $\mathrm{A}^{\prime} \mathrm{B}$ 和 $\mathrm{AB}$ 的平均剪 切模量和平均有效晶格常数; $\Delta R^{*}$ 为溶质晶胞与基 体晶胞的 $R^{*}$ 之差; $Z$ 为晶胞或其对应的超晶胞内的 结构基元数, $M$ 为无量纲常数, 约为 $7.34^{[26]}$ 。从式(3) 可以看出, 溶解度因子 $\delta$ 越小, 晶格畸变能越低, $\mathrm{A}^{\prime} \mathrm{B}$ 和 $\mathrm{AB}$ 越容易形成完全固溶体。当晶胞体积相 同时, 高对称性结构的 $R^{*}$ 往往低于低对称性晶体结 构的 $R^{*}$, 因此其溶解度因子 $\delta$ 小, 晶格畸变能低。 对于相变材料, 其高温相通常为高对称性结构, 而 其低温相通常为低对称性结构。这种高对称性结构 和低对称性结构在固溶过程中产生不同的晶格畸变 能，是构型熵增加导致材料高对称性结构向低温移 动的根本原因。

\section{2 熵与热输运性质的关联}

如图 3 所示, 材料构型熵的增加会导致晶格热 导率降低。多种元素在同一晶格位置共存时, 元素 之间的质量差异和原子半径差异, 将引入质量场涨 落和应力场涨落, 进而使溶质原子作为点缺陷散射 中心干扰高频声子的传输, 降低晶格热导率。与此 同时, 熵工程可以诱发动力学上的迟滞效应, 进而 诱导局部微观区域出现晶格畸变。这些纳米-微米尺 度的晶格畸变将与点缺陷共同作用, 在材料中形成 全尺度的多级微观结构, 对声子实现宽频散射, 大 幅降低材料的晶格热导率。理论上, 晶格热导率将 随固溶体组分增加而逐渐降低。当固溶体组分达到 一定数值时, 材料晶格热导率可以接近理论最低 值。但是, 不同材料体系达到晶格热导率理论最

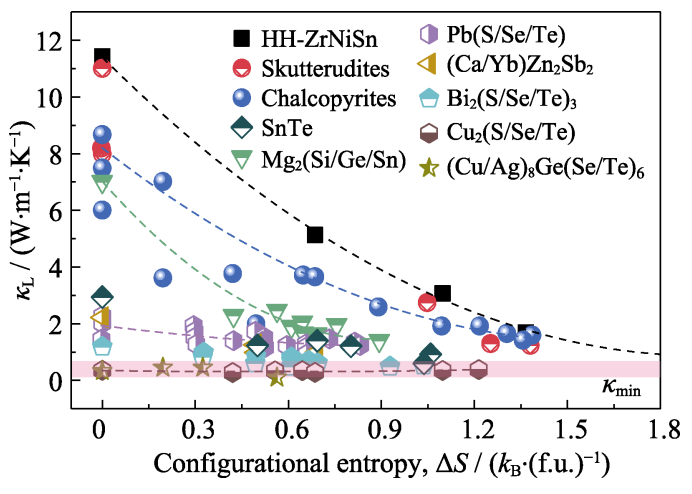

图 3 代表性热电材料晶格热导率与构型熵的关系 ${ }^{[10,39-40]}$

Fig. 3 Lattice thermal conductivity as a function of configurational entropy for typical TE materials ${ }^{[10,39-40]}$

The red zone presents the minimum lattice thermal conductivity

低值所需的固溶体组分不同。对于晶格热导率较高 的半赫斯勒合金 ${ }^{[27]}$ 、方钴矿 ${ }^{[28]}$ 、黄铜矿 ${ }^{[29]}$ 等材料体 系, 在 $300 \mathrm{~K}$ 下需要 5 6 种固溶体组分才可以使晶格 热导率达到理论最低值。例如, $\mathrm{CuInTe}_{2}{ }^{[30]}$ 或者 $\mathrm{CuGaTe}_{2}{ }^{[31]}$ 基体在室温晶格热导率 $\left(\kappa_{\mathrm{L}}\right)$ 为 (6 9) $\mathrm{W} \cdot \mathrm{m}^{-1} \cdot \mathrm{K}^{-1}$; 当固溶体组分为 2 时, 对应的构型 熵为 $0.69 k_{\mathrm{B}} /$ (f.u.), $\kappa_{\mathrm{L}}$ 降低到 $(2 \sim 4) \mathrm{W} \cdot \mathrm{m}^{-1} \cdot \mathrm{K}^{-1[31]}$; 当 固溶体组分为 4 时, 对应的构型熵为 $1.38 k_{\mathrm{B}} /($ f.u. $), \kappa_{\mathrm{L}}$ 降低到 $1.4 \mathrm{~W} \cdot \mathrm{m}^{-1} \cdot \mathrm{K}^{-1[10]}$ 。对于 $\kappa_{\mathrm{L}}$ 适中的 $(\mathrm{Ca} / \mathrm{Yb}) \mathrm{Zn}_{2} \mathrm{Sb}_{2}{ }^{[32]} 、 \mathrm{Mg}_{2}(\mathrm{Si} / \mathrm{Ge} / \mathrm{Sn})^{[33-35]} 、 \mathrm{Bi}_{2}(\mathrm{~S} / \mathrm{Se} / \mathrm{Te})_{3}{ }^{\left[{ }^{[3]}\right.} 、$

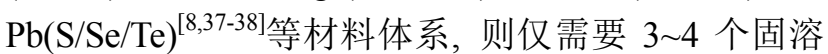
体组分， $\kappa_{\mathrm{L}}$ 即可降到理论最低值。例如, $\mathrm{PbTe}$ 基体 ${ }^{[8]}$ 的室温 $\kappa_{\mathrm{L}}$ 为 $2.5 \mathrm{~W} \cdot \mathrm{m}^{-1} \cdot \mathrm{K}^{-1}$; 当固溶体组分为 3 时, 对应的构型熵为 $0.7 k_{\mathrm{B}} /($ f.u. $), \kappa_{\mathrm{L}}$ 降 低 到 $1.0 \mathrm{~W} \cdot \mathrm{m}^{-1} \cdot \mathrm{K}^{-1[38]}$ 。而对于 $\kappa_{\mathrm{L}}$ 几乎接近于理论极限的 $\mathrm{Cu}_{2}(\mathrm{~S} / \mathrm{Se} / \mathrm{Te}) 、(\mathrm{Cu} / \mathrm{Ag})_{8} \mathrm{Ge}(\mathrm{Se} / \mathrm{Te})_{6}$ 等类液态材料体 系, 引入固溶体组分后, $\kappa_{\mathrm{L}}$ 基本维持不变 ${ }^{[10]}$ 。除增 加构型熵之外，对自旋熵、振动熵、扩散熵等调控也 有望增强材料的无序度, 进而降低晶格热导率 ${ }^{[39-40]}$ 。

\section{2 熵工程在典型热电材料体系中的应用}

目前, 熵工程已经在多个热电材料体系得到了 应用, 并实现了 $Z T$ 值的显著提升(见图 4)。本部分 将对代表性工作进行总结。

\section{1 类液态材料体系}

2012 年, Liu 等 ${ }^{[9]}$ 提出在固态材料中引入具有 “类液态”特征的离子来降低晶格热导率和优化热电 性能的设计策略，成功突破了晶格热导率在固态玻 璃或晶态材料上的限制，进而发现了一大类具有 


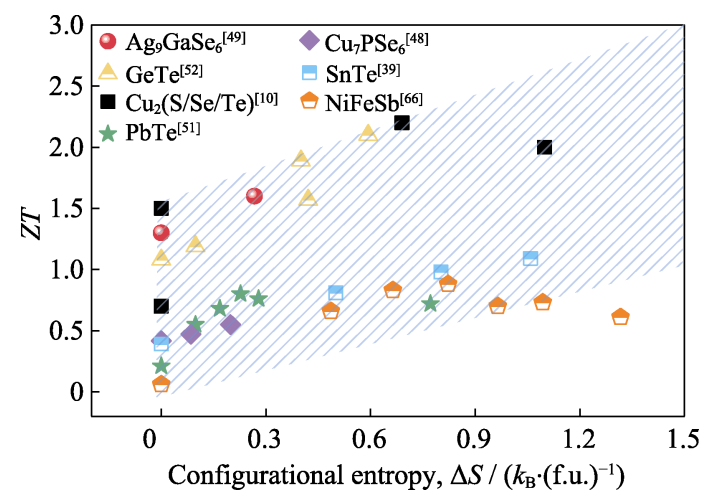

图 4 代表性热电材料体系中 $Z T$ 值与构型熵的关系

Fig. 4 TE figure of merit as a function of configurational entropy for typical TE materials

“声子液体一电子晶体”特征的新型高性能类液态热 电材料体系。类液态热电材料体系是熵工程这一电 热输运解耦新方法最早的应用对象。区别于传统块 体热电材料, 类液态热电材料一般具有至少一个温 度相关的相变。相变之前, 类液态热电材料具有诸 如单斜、三斜、正交等低对称性的晶体结构; 而相 变之后, 类液态热电材料将转变为具有诸如六方或 立方等高对称性的晶体结构, 其中阳离子激活能很 低，可以在阴离子或阴离子基团构成的框架中随机 迁移, 从而导致极低的本征晶格热导率 ${ }^{[9]}$ 。熵工程在 类液态热电材料体系中的作用主要体现在降低相变 温度, 提高低温下晶体结构的对称性, 进而优化电 输运性能。

$\mathrm{Cu}_{2} \mathrm{X}(\mathrm{X}=\mathrm{S}, \mathrm{Te}, \mathrm{Se})$ 是一类典型的类液态热电 材料。 $\mathrm{Cu}_{2} \mathrm{~S}$ 在 $376 \mathrm{~K}$ 以下呈低对称单斜结构(空间 群: $\mathrm{P} 2_{1} / \mathrm{c}$ ); 在 376 710 K 呈高对称性六方结构(空间 群: $\mathrm{P} 6_{3} / \mathrm{mmc}$ ); 在 $710 \mathrm{~K}$ 以上呈具有更高对称性的立 方结构(空间群: $\mathrm{Fm} \overline{3} \mathrm{~m})^{[41]}$ 。类似地, $\mathrm{Cu}_{2} \mathrm{Se}$ 和 $\mathrm{Cu}_{2} \mathrm{Te}$ 随温度升高也会从低对称性结构向高对称性结构发 生转变 ${ }^{[42-43]}$ 。研究发现, 通过在 $\mathrm{Cu}_{2} \mathrm{~S}$ 中固溶 $\mathrm{Se}$ 和 (或) Te 提高构型熵, 可以将单斜 $\rightarrow$ 六方结构相变温 度从 $376 \mathrm{~K}$ 显著降低至室温以下。例如, $\mathrm{Cu}_{2} \mathrm{~S}_{0.5} \mathrm{Te}_{0.5}{ }^{[44]} 、 \mathrm{Cu}_{2} \mathrm{~S}_{0.5} \mathrm{Se}_{0.5}{ }^{[45]}$ 和 $\mathrm{Cu}_{2} \mathrm{~S}_{1 / 3} \mathrm{Se}_{1 / 3} \mathrm{Te}_{1 / 3}{ }^{[46]}$ 室温下即呈高对称性六方结构。晶体结构对称性的 提高使得在倒空间中等效位点增加, 能带简并度增 强, 从而提高材料的态密度有效质量 $m$ * 和泽贝克 系数。如图 5 所示, $\mathrm{Cu}_{2} \mathrm{~S} 、 \mathrm{Cu}_{2} \mathrm{Se}$ 和 $\mathrm{Cu}_{2} \mathrm{Te}$ 低对称性 单斜结构的 $m$ *均小于 $2 m_{\mathrm{e}}$ ( $m_{\mathrm{e}}$ 为电子有效质量)。但 是，通过熵工程，具有高对称六方相的 $\mathrm{Cu}_{2} \mathrm{~S}_{0.5} \mathrm{Te}_{0.5}$ 、 $\mathrm{Cu}_{2} \mathrm{~S}_{0.5} \mathrm{Se}_{0.5}$ 和 $\mathrm{Cu}_{2} \mathrm{~S}_{1 / 3} \mathrm{Se}_{1 / 3} \mathrm{Te}_{1 / 3}$ 的 $m^{*}$ 增大至 $4.5 m_{\mathrm{e}}$ 。 由于晶体结构对称性的增加, 在同样载流子浓度下 泽贝克系数得到显著增强。例如, 当载流子浓度在 $(1 \sim 3) \times 10^{21} \mathrm{~cm}^{-3}$ 范围时，六方结构的 $\mathrm{Cu}_{2} \mathrm{~S}_{0.5} \mathrm{Te}_{0.5}$ 、

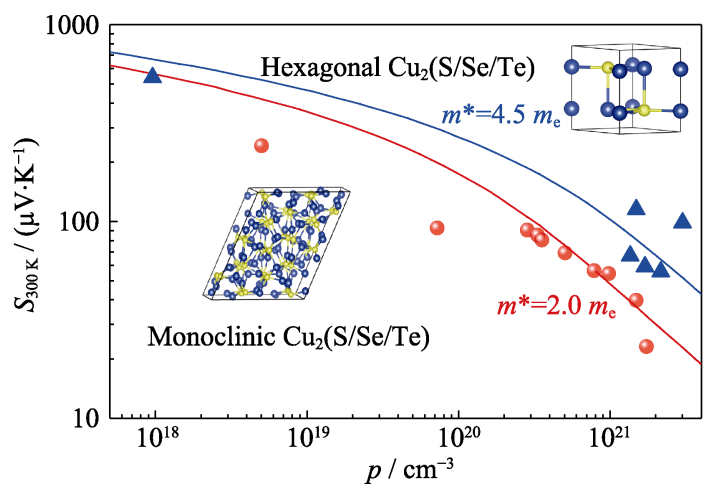

图 $5 \mathrm{Cu}_{2} \mathrm{X}(\mathrm{X}=\mathrm{S}, \mathrm{Te}, \mathrm{Se})$ 体系泽贝克系数与载流子浓度的关系 ${ }^{[10]}$ Fig. 5 Carrier concentration dependence of room-temperature Seebeck coefficient in $\mathrm{Cu}_{2}(\mathrm{~S} / \mathrm{Se} / \mathrm{Te})$-based $\mathrm{TE}$ materials with different crystal symmetry ${ }^{[10]}$

$\mathrm{Cu}_{2} \mathrm{~S}_{0.5} \mathrm{Se}_{0.5}$ 和 $\mathrm{Cu}_{2} \mathrm{~S}_{1 / 3} \mathrm{Se}_{1 / 3} \mathrm{Te}_{1 / 3}$ 室温下泽贝克系数在 (70 130) $\mu \mathrm{V} / \mathrm{K}$ 之间，远高于单斜结构的 $\mathrm{Cu}_{2} \mathrm{X}(\mathrm{X}=$ $\mathrm{Te}, \mathrm{Se}, \mathrm{S})$ 的泽贝克系数 $((20 \sim 40) \mu \mathrm{V} / \mathrm{K})$ 。最终, $\mathrm{Cu}_{2} \mathrm{~S}_{0.5} \mathrm{Te}_{0.5}$ 和 $\mathrm{Cu}_{2} \mathrm{~S}_{1 / 3} \mathrm{Se}_{1 / 3} \mathrm{Te}_{1 / 3}$ 的 $Z T$ 在 $1000 \mathrm{~K}$ 时接 近 2.0, 较 $\mathrm{Cu}_{2} \mathrm{~S}$ 提高近 2 倍 ${ }^{[10]}$ 。

熵工程在硫银锗矿型热电化合物中也得到了成 功应用。 $\mathrm{Cu}_{7} \mathrm{PSe}_{6}$ 是热电领域最早被关注的硫银锗 矿型化合物之一 ${ }^{[47]}$ 。 $\mathrm{Cu}_{7} \mathrm{PSe}_{6}$ 在 $250 \mathrm{~K}$ 以下呈低对 称的正交结构, 320 250 K 呈简单立方结构, $320 \mathrm{~K}$ 以上呈高对称的面心立方结构。研究发现, 通过在 $\mathrm{Se}$ 位固溶 $\mathrm{Te}$ 增加构型熵, 可以显著降低 $\mathrm{Cu}_{7} \mathrm{PSe}_{6}$ 相变温度。当 $\mathrm{Te}$ 固溶量为 0.3 时, 即使在 $150 \mathrm{~K}$ 低 温下，材料仍然具有高对称的面心立方结构。由于 晶格对称性的提高, 材料的室温 $m *$ 从 $0.13 m_{\mathrm{e}}$ 增大 到 $2.0 \mathrm{~m}_{\mathrm{e}}$, 但其载流子迁移率并未发生明显变化。当 载流子浓度在 $1.0 \times 10^{21} \mathrm{~cm}^{-3}$ 左右时, $\mathrm{Cu}_{7} \mathrm{PSe}_{5.7} \mathrm{Te}_{0.3}$ 高对称面心立方结构的泽贝克系数为 $130 \mu \mathrm{V} / \mathrm{K}$, 约 为 $\mathrm{Cu}_{7} \mathrm{PSe}_{6}$ 低对称简单立方相的 3 倍 ${ }^{[48]}{ }^{\circ} \mathrm{Ag}_{9} \mathrm{GaSe}_{6}$ 硫银锗矿型热电化合物是目前 $Z T$ 值最高的 $\mathrm{n}$ 型类液 态热电材料, 它在 $281 \mathrm{~K}$ 以下呈简单立方结构, $281 \sim 287 \mathrm{~K}$ 呈低对称三方结构, 在 $287 \mathrm{~K}$ 以上呈高对 称面心立方结构。Jiang 等 ${ }^{[49]}$ 在 $\mathrm{Ag}_{9} \mathrm{GaSe}_{6}$ 的 $\mathrm{Se}$ 位固 溶 $\mathrm{Te}$ 位以增加构型熵，成功消除了 281 287 K 区间 的三方结构。因为 $\mathrm{Ag}_{9} \mathrm{GaSe}_{6}$ 在室温已经具有极低的 晶格热导率, 所以熵工程对其室温以上的晶格热导 率的降低作用不明显，但是，熵工程可将其晶格热 导率从 $12 \mathrm{~K}$ 的 $1.1 \mathrm{~W} \cdot \mathrm{m}^{-1} \cdot \mathrm{K}^{-1}$ 降低至 $0.6 \mathrm{~W} \cdot \mathrm{m}^{-1} \cdot \mathrm{K}^{-1}$ 。 在 $850 \mathrm{~K}$ 时, 构型熵为 $0.26 k_{\mathrm{B}}$ (f.u.)的 $\mathrm{Ag}_{9} \mathrm{GaSe}_{5.53} \mathrm{Te}_{0.45}$ 最大 $Z T$ 值约为 1.6 , 与 $\mathrm{Ag}_{9} \mathrm{GaSe}_{6}$ 基体相比提高了 $45 \%{ }^{[50]}$ 。

\section{$2.2 \mathrm{IV}-\mathrm{VI}$ 族化合物}

IV-VI族半导体(包括 PbTe、GeTe、SnSe、SnTe、 
$\mathrm{SnS}$ 等化合物)是一类非常重要的热电材料 ${ }^{[51-53]}$ 。近 年来, 熵工程成功地应用于 IV - VI 族半导体热电性 能的优化。Hu 等 ${ }^{[39]}$ 通过在 $\mathrm{SnTe}$ 中的 $\mathrm{Sn}$ 位引入 $\mathrm{Ge}$ 、 $\mathrm{Pb} 、 \mathrm{Mn}$ 多种元素来提高构型摘, 发现热力学上的高 熵效应使得 $\mathrm{Mn}$ 的固溶度极限从二元 $\mathrm{SnTe}$ 体系中的 (13 15)mol\%显著增大至四元 $\left(\mathrm{Sn}_{0.7} \mathrm{Ge}_{0.2} \mathrm{~Pb}_{0.1}\right) \mathrm{Te}$ 体 系中的 $20 \mathrm{~mol} \%$ 。高构型熵的 $(\mathrm{Sn}, \mathrm{Ge}, \mathrm{Pb}, \mathrm{Mn}) \mathrm{Te}$ 体 系具有高的能带简并度, 使其在相同载流子浓度下 具有比二元 $\mathrm{SnTe}$ 高的泽贝克系数 (见图 6(a))。与此 同时, 在多元素所形成的原子尺度点缺陷、线缺陷, 纳米尺度的应变簇和微米尺度的界面多种因素共同 作用下, SnTe 化合物在 $900 \mathrm{~K}$ 的晶格热导率从 $1.6 \mathrm{~W} \cdot \mathrm{m}^{-1} \cdot \mathrm{K}^{-1}$ 显著降低至 $0.5 \mathrm{~W} \cdot \mathrm{m}^{-1} \cdot \mathrm{K}^{-1}$ (见图 6(b)), 接近理论最低极限值。构型摘为 $1.15 \mathrm{k}_{\mathrm{B}}$ /(f.u.) 的 $\left(\mathrm{Sn}_{0.74} \mathrm{Ge}_{0.2} \mathrm{~Pb}_{0.1}\right)_{0.75} \mathrm{Mn}_{0.275} \mathrm{Te}$ 在 $900 \mathrm{~K}$ 时 $\mathrm{ZT}$ 达 1.42, 相对于 $\mathrm{SnTe}$ 基体提升约 3 倍 ${ }^{[39]}$ 。Zhao 等 ${ }^{[40]}$ 合成了 具有高构型熵的 $\left(\mathrm{Sn}_{0.5} \mathrm{Ge}_{0.4875}\right)_{1-x} \mathrm{~Pb}_{x} \mathrm{Te}$ 化合物, 发现 其室温下的晶格热导率由 $1.4 \mathrm{~W} \cdot \mathrm{m}^{-1} \cdot \mathrm{K}^{-1}(x=0)$ 降低 至 $0.6 \cdot \mathrm{W} \cdot \mathrm{m}^{-1} \cdot \mathrm{K}^{-1}(x=0.5),\left(\mathrm{Sn}_{0.5} \mathrm{Ge}_{0.4875}\right)_{0.5} \mathrm{~Pb}_{0.5} \mathrm{Te}$ 在 $773 \mathrm{~K}$ 时最大 $Z T$ 达到 1.61 。相比于在一个原子位置 进行调控, 在两个原子位置引入多组分元素改变构 型熵, 对晶格热导率的降低作用更加显著。对于
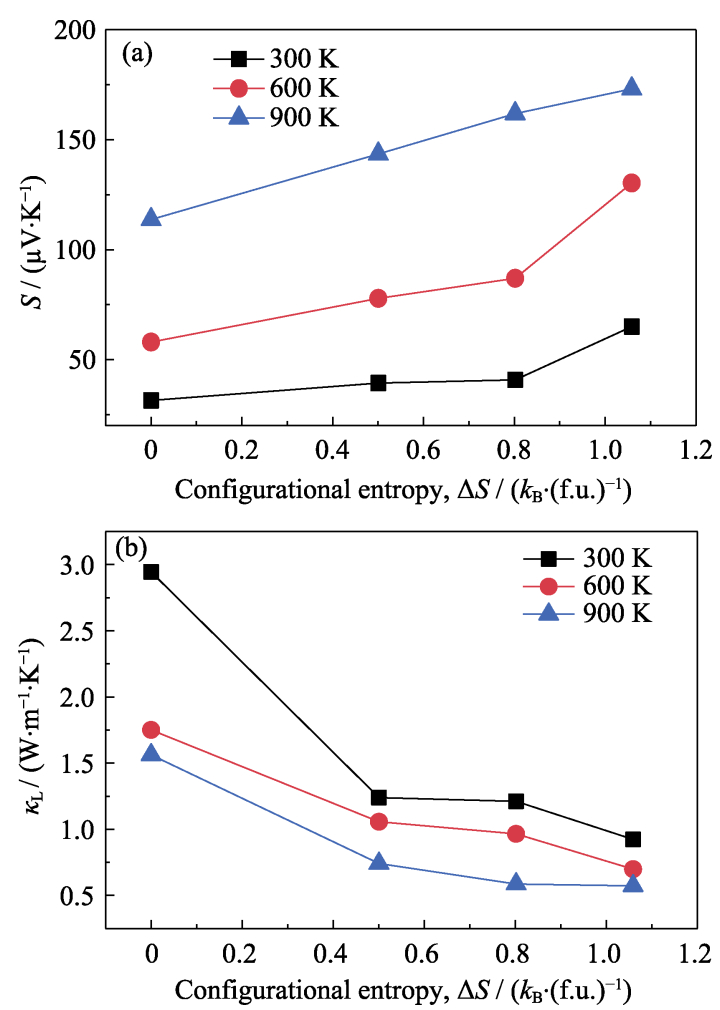

图 $6(\mathrm{Sn}, \mathrm{Ge}, \mathrm{Pb}, \mathrm{Mn}) \mathrm{Te}$ 体系(a)泽贝克系数和(b)晶格热导 率与构型熵的关系 ${ }^{[39]}$

Fig. 6 (a) Seebeck coefficient and (b) lattice thermal conductivity as a function of configurational entropy in (Sn, Ge, $\mathrm{Pb}, \mathrm{Mn})$ Te-based materials ${ }^{[39]}$
PbSnTeSe 化合物, 其室温晶格热导率显著低于 $\mathrm{PbTe}-\mathrm{PbSe} 、 \mathrm{PbTe}-\mathrm{PbSe}-\mathrm{PbS}$ 等固溶体 ${ }^{[54]}$ 。 $\mathrm{Wu}$ 等 ${ }^{[55]}$ 在 $\mathrm{PbTe}$ 材料中的 $\mathrm{Pb}$ 位引入 $\mathrm{Na} 、 \mathrm{Mn} 、 \mathrm{Eu}$ 元素掺杂, 由于构型熵的增加, 在相近载流子浓度下, 材料室 温泽贝克系数从 $80 \mu \mathrm{V} / \mathrm{K}$ 增大到 $130 \mu \mathrm{V} / \mathrm{K}$, 其中 $\mathrm{Na}_{0.03} \mathrm{Eu}_{0.03} \mathrm{Mn}_{0.03} \mathrm{~Pb}_{0.91} \mathrm{Te}$ 在 $850 \mathrm{~K}$ 下的 $Z T$ 达到了 2.4。 部分IV-VI族半导体(如 GeTe、GeSe、SnSe 等) 也具有与温度相关的相变 ${ }^{[6-58]}$ 。类似于上述类液态 热电材料体系, 通过熵工程也可以影响其相变特征。 在 $930 \mathrm{~K}$ 左右, $\mathrm{GeSe}$ 晶体结构从低对称正交结构转 变为高对称立方结构 ${ }^{[59-60]}$ 。Huang 等 ${ }^{[61]}$ 通过在 $\mathrm{GeSe}$ 中引入 $\mathrm{AgSbSe}_{2}$ 增加构型熵, 发现其从室温下正交 结构转变为具有更高对称性的菱方结构。与此同时, 材料的立方相转变温度也由 $930 \mathrm{~K}$ 降低至 $523 \mathrm{~K}$ 。由 于室温下晶体结构对称性的提高, 材料的态密度有 效质量从 $0.75 \mathrm{~m}_{\mathrm{e}}$ 显著增大至 $1.8 \mathrm{~m}_{\mathrm{e}}$ 。结合载流子浓 度的优化和晶格热导率的降低, 材料的热电性能显 著提升。 $710 \mathrm{~K}, \mathrm{GeAg}_{0.2} \mathrm{Sb}_{0.2} \mathrm{Se}_{1.4}$ 的 $\mathrm{ZT}$ 值达到 0.86 , 较 $\mathrm{GeSe}$ 基体提高约 18 倍。此外, $660 \mathrm{~K}$ 左右, $\mathrm{GeTe}$ 的晶体结构从低对称菱方结构转变为高对称立方结 构。Qiu 等 ${ }^{[62]}$ 在 $\mathrm{GeTe}$ 化合物中引入 $\mathrm{In} 、 \mathrm{~Pb} 、 \mathrm{Sb} 、 \mathrm{I}$ 多种组分元素增加构型熵, 在 $523 \mathrm{~K}$ 即实现低对称菱 方结构向高对称立方结构的转变。构型熵的增加显 著提高了态密度有效质量, 使室温泽贝克系数从 $39 \mu \mathrm{V} / \mathrm{K}$ ( $\mathrm{GeTe}$ 二元化合物) 提升至 $140 \mu \mathrm{V} / \mathrm{K}$ $\left(\mathrm{Ge}_{0.84} \mathrm{In}_{0.010} \mathrm{~Pb}_{0.1} \mathrm{Sb}_{0.05} \mathrm{Te}_{0.997} \mathrm{I}_{0.003}\right)$ 。与此同时, 构型 熵为 $0.59 k_{\mathrm{B}} /($ f.u. $)$ 的 $\mathrm{Ge}_{0.84} \mathrm{In}_{0.010} \mathrm{~Pb}_{0.1} \mathrm{Sb}_{0.05} \mathrm{Te}_{0.997} \mathrm{I}_{0.003}$ 化合物的晶格热导率降低至 $0.4 \mathrm{~W} \cdot \mathrm{m}^{-1} \cdot \mathrm{K}^{-1}$, 接近根 据 Cahill 模型计算得到的理论最低极限值。最终, $\mathrm{Ge}_{0.84} \mathrm{In}_{0.010} \mathrm{~Pb}_{0.1} \mathrm{Sb}_{0.05} \mathrm{Te}_{0.997} \mathrm{I}_{0.003}$ 在 $800 \mathrm{~K}$ 的 $\mathrm{ZT}$ 值达 到 2.1。

\section{3 其它材料体系}

除上述类液态材料和 IV - VI 族半导体之外，熵 工程在类金刚石化合物、 $\mathrm{Bi}_{2} \mathrm{Te}_{3}$ 、半赫斯勒合金等 材料体系也得到了应用, 其作用主要体现在降低晶 格热导率。 $\mathrm{Liu}$ 等 ${ }^{[10]}$ 合成了具有高构型熵的类金刚 石化合物 $\mathrm{Cu}_{0.5} \mathrm{Ag}_{0.5} \mathrm{In}_{0.5} \mathrm{Ga}_{0.5} \mathrm{Te}_{2}$, 其在室温下晶格热 导率仅为 $1.8 \mathrm{~W} \cdot \mathrm{m}^{-1} \cdot \mathrm{K}^{-1}$, 较 $\mathrm{CuInTe}_{2}$ 或者 $\mathrm{CuGaTe}_{2}$ 基体 $\left(\kappa_{\mathrm{L}}=(6 \sim 9) \mathrm{W} \cdot \mathrm{m}^{-1} \cdot \mathrm{K}^{-1}\right)$ 显著降低。Zhang 等 ${ }^{[63]}$ 通 过大数据分析篮选并制备了 $\mathrm{Cu}$ 基类金刚石型高熵热 电材料 $\mathrm{Cu}_{5} \mathrm{SnMgGeZnS}_{9}$, 它在 $773 \mathrm{~K}$ 的晶格热导率 仅为 $0.4 \mathrm{~W} \cdot \mathrm{m}^{-1} \cdot \mathrm{K}^{-1}$, 显著低于传统三元 $\mathrm{Cu}$ 基类金刚 石材料 $\mathrm{CuGaTe}_{2}$ 和 $\mathrm{CuInTe}_{2}$ 。Fan 等 ${ }^{[64]}$ 在 $\mathrm{Bi}_{2} \mathrm{Te}_{3}$ 化合 物的 $\mathrm{Bi}$ 和 $\mathrm{Te}$ 原子位置分别加入 $\mathrm{Sb}$ 和 $\mathrm{Se}$ 增加构型 熵, $400 \mathrm{~K}$ 时其晶格热导率降低至 $0.47 \mathrm{~W} \cdot \mathrm{m}^{-1} \cdot \mathrm{K}^{-1}$ 。 
Raphel 等 ${ }^{[65]}$ 采用高能球磨结合放电等离子烧结制 备了具有纳米结构的 $\mathrm{BiSbTe}_{1.5} \mathrm{Se}_{1.5}$, 在熵工程和晶 界的共同作用下，其晶格热导率在 $523 \mathrm{~K}$ 仅为 $0.2 \mathrm{~W} \cdot \mathrm{m}^{-1} \cdot \mathrm{K}^{-1}$, 接近理论最低极限值。Yan 等 ${ }^{[66]}$ 采 用电弧熔炼法制备了 $\mathrm{Nb}_{1-x} \mathrm{M}_{x} \mathrm{FeSb}(\mathrm{M}=\mathrm{Hf}, \mathrm{Zr}, \mathrm{Mo}$, $\mathrm{V}, \mathrm{Ti})$ 半赫斯勒高熵热电材料, 由于在 $\mathrm{Nb}$ 亚晶格中 引入多种组分, 材料的构型熵显著增加, 导致其晶 格热导率大幅度降低, 在 $800 \mathrm{~K}$ 时, 晶格热导率从 $5.6 \mathrm{~W} \cdot \mathrm{m}^{-1} \cdot \mathrm{K}^{-1}\left(\mathrm{NbFeSb}\right.$ 基体) 降低到 $2.5 \mathrm{~W} \cdot \mathrm{m}^{-1} \cdot \mathrm{K}^{-1}$ $\left(\mathrm{Nb}_{0.6} \mathrm{M}_{0.4} \mathrm{FeSb}(\mathrm{M}=\mathrm{Hf}, \mathrm{Zr}, \mathrm{Mo}, \mathrm{V}, \mathrm{Ti})\right)$ 。

\section{3 多元单相高熵热电材料稳定性预测}

\section{方法}

熵工程已经被证明是一种有效的实现热电材料 电热输运性质解耦的新方法。但是, 熵工程的应用 所面临的最大问题在于具有高构型熵的稳定单相材 料的制备。由于晶格畸变能的增加, 引入多种组元 在增加构型熵的同时也会增加材料的焓, 从而造成 材料趋于不稳定。例如, 二元体系 $\mathrm{PbTe}$ 与 $\mathrm{PbS}, \mathrm{PbTe}$ 和 $\mathrm{GeTe}$ 均很难形成高固溶度的稳定单相固溶体。 而对于一个原子位置同时存在 3 种及以上元素的多 组元体系, 情况更为复杂, 依靠传统的实验试错方 法制备单相固溶体需要耗费大量人力、时间和物力。 因此, 为了更广泛地应用熵工程, 迫切需要开发有 效的多元单相高熵热电材料稳定性预测方法。

在多元热电材料体系中, 构型摘取决于体系中 的固溶元素种类和各自的固溶度, 而能否形成具有 一定固溶元素种类和固溶度的关键在于体系的固溶 焓与构型熵之间的竞争。吉布斯自由能小于 0 是多 元单相高熵热电材料形成的必要前提。固溶过程中 的吉布斯自由能的表达式为 ${ }^{[23]}$ :

$$
\Delta G_{\text {mix }}=\Delta H_{\text {mix }}-T \Delta S_{\text {mix }}<0
$$

其中, $\Delta H_{\mathrm{mix}}$ 为固溶过程中焓的变化, $\Delta S_{\mathrm{mix}}$ 为固溶 过程中熵的变化。 $\Delta S_{\text {mix }}$ 可以通过方程(1)得到。Chen 根据弹性力学模型 ${ }^{[25]}$, 在二元固溶体晶格畸变能 (方程(3))的基础上, 进一步得到了 $n$ 元完全固溶体 的晶格畸变能 ${ }^{[10]}$ :

$$
\Delta \bar{H}_{\text {total }}=M N_{\mathrm{A}} \cdot \bar{\delta} \cdot\left[\sum_{i=2}^{n}\left(1-\frac{1}{i}\right) \frac{1}{i} \cdot\left(\frac{i}{n}\right)^{3.5}\right]
$$

其中, $\bar{\delta}$ 为所有基体的平均 $\delta$ 因子。进而, 材料完全 固溶时的吉布斯自由能表达式为 ${ }^{[10]}$ :

$$
\Delta G_{\text {mix }}=M N_{\mathrm{A}} \cdot \bar{\delta} \cdot\left[\sum_{i=2}^{n}\left(1-\frac{1}{i}\right) \frac{1}{i} \cdot\left(\frac{i}{n}\right)^{3.5}\right]-N_{\mathrm{A}} k_{\mathrm{B}} T \ln n
$$

根据方程(6), 材料完全固溶的吉布斯自由能仅与材 料的平均溶解度参数 $\bar{\delta}$ 和材料的组元数 $n$ 有关。如 图 7 所示, $400 \mathrm{~K}$ 形成二元单相完全固溶体， $\bar{\delta}$ 因子 需要小于 $2.08 \mathrm{GPa} \cdot \AA^{3}(1 \AA=0.1 \mathrm{~nm})$; 形成三元单相 完全固溶体, $\bar{\delta}$ 因子需要小于 $2.92 \mathrm{GPa} \cdot \AA^{3}$; 形成四 元单相完全固溶体， $\bar{\delta}$ 因子需要小于 $3.58 \mathrm{GPa} \cdot \AA^{3}$ 。

根据方程(6), $\Delta G_{\mathrm{mix}}$ 随组元数 $n$ 增加而逐渐降 低。图 8 所示为不同材料体系的焓变与组元数的关 系, 其中黑线代表完全固溶时的熵变: $T \Delta S=$ $N_{\mathrm{A}} k_{\mathrm{B}} T \ln n$ 。该线下方区域表示熵变能量大于焓变, $\Delta G_{\mathrm{mix}}$ 小于 0 , 材料能够完全固溶; 该线上方区域表 示熵变能量小于焓变, $\Delta G_{\mathrm{mix}}$ 大于 0 , 材料不能完全 固溶。因此, 对于 $\bar{\delta}$ 因子较小的材料体系, 如 $(\mathrm{Ti} / \mathrm{Zr} / \mathrm{Hf}) \operatorname{CoSb}$, 仅需要 $n=2$ 就可以获得单相的固溶 体 ${ }^{[67]}$; 对于 $\bar{\delta}$ 因子较大的材料体系, 如 $\mathrm{Pb}(\mathrm{S} / \mathrm{Se} / \mathrm{Te})$, 至少需要 $n=5$ 才可以获得单相的固溶体 ${ }^{[68]}$; 而对 $\bar{\delta}$ 因子非常大的材料体系, 如 $\mathrm{Mg}_{2}(\mathrm{Si} / \mathrm{Ge} / \mathrm{Sn})$, 则需要 更高的组元数才可以得到单相固溶体。相关理论预

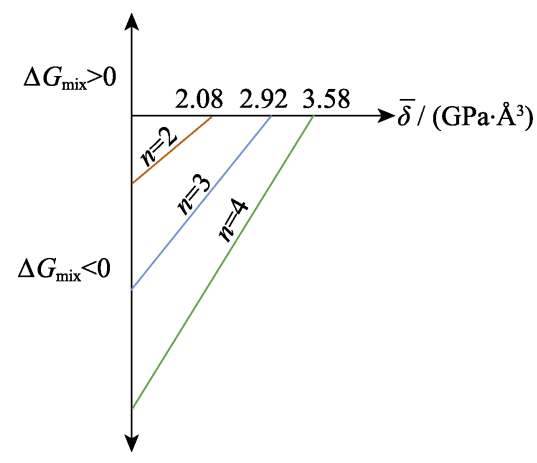

图 7 固溶吉布斯自由能与平均溶解度参数 $\bar{\delta}$ 和材料组元 数 $n$ 的关系 ${ }^{[10]}(1 \AA=0.1 \mathrm{~nm})$

Fig. 7 Gibbs free energy as a function of the average solubility parameter $(\bar{\delta})$ for given multicomponent TE materials with different number of components ${ }^{[10]}(1 \AA=0.1 \mathrm{~nm})$

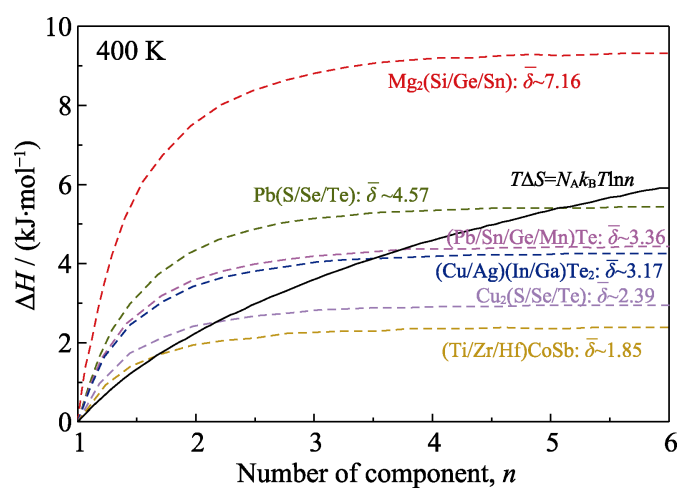

图 8 不同材料体系多元固溶焓变与组元数 $n$ 的关系 ${ }^{[10]}$ Fig. 8 Enthalpy as a function of the number of components for typical TE materials ${ }^{[10]}$ 
测结果与实验现象完全吻合。在这个多元单相高熵 热电材料稳定性预测方法的指引下, Liu 等 ${ }^{[10]}$ 成功 制备了 $\mathrm{Cu}_{2}\left(\mathrm{~S}_{1 / 3} \mathrm{Se}_{1 / 3} \mathrm{Te}_{1 / 3}\right)$ 和 $\mathrm{Cu}_{0.5} \mathrm{Ag}_{0.5} \mathrm{In}_{0.5} \mathrm{Ga}_{0.5} \mathrm{Te}_{2}$ 两 种单相高熵热电材料。

\section{4 总结与展望}

作为高熵合金设计思想的延伸, 熵工程可从电 子和声子输运两方面指引热电材料的性能优化, 在 类液态材料、半赫斯勒合金、IV-VI 族化合物等多 种热电材料体系已经获得了成功应用。通过增加构 型商, 可以同时提高泽贝克系数和降低晶格热导率, 进而显著优化热电性能。本文讨论了构型熵提高泽 贝克系数和降低热导率的具体物理机制, 并选取了 一系列典型的热电材料展现如何应用熵工程用以提 升材料的热电性能。特别需要指出的是, 由于熵这 一基础参量具有内禀的类似基因特性, 它可以作为 热电材料的性能指征因子, 对多元热电材料实现快 速篮选, 体现了材料基因工程研究方法在热电材料 上的应用，丰富了“材料基因工程”理念的研究领域 和内容。

熵工程虽然已经在诸多热电材料体系中得到了 应用，但是仍处于起步阶段。根据多元单相高熵热 电材料稳定性预测方法, 从理论上设计开发热力学 稳定的新型高性能热电材料体系将是熵工程的重点 研究方向之一。与此同时, 依据每个材料体系自身 特点, 进一步细化熵对电子和声子输运的影响, 有 针对性地设计最佳固溶元素种类、数目、固溶量, 寻 找对载流子和声子散射的最佳平衡点, 在尽可能提 高泽贝克系数和降低晶格热导率的同时尽量减小对 载流子迁移率的影响, 是熵工程应用的必经之路。

此外, 当前熵工程主要集中于增加构型熵, 而 对自旋熵 ${ }^{[69]}$ 、振动摘 ${ }^{[70]}$ 、扩散摘 ${ }^{[71]}$ 等调控也有望影 响电热输运性质进而提升热电优值。例如, Wang 等 ${ }^{[69]}$ 发现由于 Co 低自旋态和高自旋态的共存而导致的 高自旋熵是 $\mathrm{Na}_{x} \mathrm{Co}_{2} \mathrm{O}_{4}$ 体系具有高泽贝克系数的主 要原因。 $\mathrm{Emin}^{[70]}$ 认为局域化的载流子诱导的振动软 化可以导致碳化硼体系泽贝克系数显著增加。Han 等 ${ }^{[71]}$ 通过离子的扩散熵与氧化还原电对反应熵的 协同效应在准固态离子凝胶中实现了高达 $17 \mathrm{mV} / \mathrm{K}$ 的巨热电势效应。但是, 相关研究仍处于起步阶段。 因此, 在构型熵的研究基础上, 进一步探索自旋熵、 振动熵、扩散熵等对材料热电性能的影响及优化, 是拓展熵工程应用的一条新途径。

再者，电热输运性能的新型“鸡尾酒”效应，结 合对微观结构的多尺度调控, 探索电声耦合新机制,
提出电热输运协同调控新方法, 也是摘工程未来的 发展方向之一。

\section{参考文献:}

[1] 陈立东, 刘睿恒, 史迅. 热电材料与器件, 北京: 科学出版社. 2018: 1-6.

[2] ZHU T J, LIU Y T, FU C G, et al. Compromise and synergy in high-efficiency thermoelectric materials. Advanced Materials, 2017, 29(14): 26

[3] SLACK G A, ROWE D. CRC Handbook of Thermoelectrics. Boca Raton, FL: CRC Press, 1995: 407-440.

[4] HICKS L, DRESSELHAUS. Thermoelectric figure of merit of a one-dimensional conductor. Physical Review B, 1993, 47(24): 16631.

[5] SHI X, ZHANG W, CHEN L D, et al. Filling fraction limit for intrinsic voids in crystals: doping in skutterudites. Physical Review Letters, 2005, 95(18): 185503.

[6] SHI X, KONG H, LI C P, et al. Low thermal conductivity and high thermoelectric figure of merit in n-type $\mathrm{Ba}_{x} \mathrm{Yb}_{y} \mathrm{Co}_{4} \mathrm{Sb}_{12}$ double-filled skutterudites. Applied Physics Letters, 2008, 92(18): 182101.

[7] SHI X, YANG J, SALVADOR J R, et al. Multiple-filled skutterudites: high thermoelectric figure of merit through separately optimizing electrical and thermal transports. Journal of the American Chemical Society, 2011, 133(20): 7837-7846.

[8] PEI Y, SHI X, LALONDE A, et al. Convergence of electronic bands for high performance bulk thermoelectrics. Nature, 2011, 473(7345): 66-69.

[9] LIU H, SHI X, XU F, et al. Copper ion liquid-like thermoelectrics. Nature Materials, 2012, 11(5): 422-425.

[10] LIU R, CHEN H, ZHAO K, et al. Entropy as a gene-like performance indicator promoting thermoelectric materials. Advanced Materials, 2017, 29(38): 1702712.

[11] YEH J W, CHEN S K, LIN S J, et al. Nanostructured high-entropy alloys with multiple principal elements: novel alloy design concepts and outcomes. Advanced Engineering Materials, 2004, 6(5): 299-303.

[12] SENKOV O N, MILLER J D, MIRACLE D B, et al. Accelerated exploration of multi-principal element alloys with solid solution phases. Nature Communications, 2015, 6(1): 6529.

[13] ZHANG Y, ZUO T T, TANG Z, et al. Microstructures and properties of high-entropy alloys. Progress in Materials Science, 2014, 61: 1-93.

[14] WEI P C, LIAO C N, WU H J, et al. Thermodynamic routes to ultralow thermal conductivity and high thermoelectric performance. Advanced Materials, 2020, 32(12): 1906457.

[15] MIRACLE D B, MILLER J D, SENKOV O N, et al. Exploration and development of high entropy alloys for structural applications. Entropy, 2014, 16(1): 494-525.

[16] ZHANG Y, ZUO T T, CHENG Y Q, et al. High-entropy alloys with high saturation magnetization, electrical resistivity, and malleability. Scientific Reports, 2013, 3: 1455.

[17] LUCAS M S, BELYEA D, BAUER C, et al. Thermomagnetic analysis of $\mathrm{FeCoCr}_{x} \mathrm{Ni}$ alloys: magnetic entropy of high-entropy alloys. Journal of Applied Physics, 2013, 113(17): 17A923.

[18] KOZELJ P, VRTNIK S, JELEN A, et al. Discovery of a superconducting high-entropy alloy. Physical Review Letters, 2014, 113(10): 107001 .

[19] KAO Y F, CHEN S K, SHEU J H, et al. Hydrogen storage properties of multi-principal-component $\mathrm{CoFeMnTi}_{x} \mathrm{~V}_{y} \mathrm{Zr}_{z}$ alloys. International Journal of Hydrogen Energy, 2010, 35(17): 9046-9059.

[20] BERARDAN D, FRANGER S, DRAGOE D, et al. Colossal dielectric constant in high entropy oxides. Physica Status Solidi-Rapid Research Letters, 2016, 10(4): 328-333.

[21] BERARDAN D, FRANGER S, MEENA A K, et al. Room temperature lithium superionic conductivity in high entropy oxides. Journal of Materials Chemistry A, 2016, 4(24): 9536-9541.

[22] SHAFEIE S, GUO S, HU Q, et al. High-entropy alloys as high-temperature thermoelectric materials. Journal of Applied Physics, 2015, 118(18): 184905.

[23] SWALIN R A. Thermodynamics of Solids. New York: John Wiley and Sons, 1972: 178 
[24] SONOMURA H. Internal strain energy in quaternary III-V compound alloys. Journal of Applied Physics, 1986, 59(3): 739-742.

[25] SLAUGHTER W, PETROLITO J. The linearized theory of elasticity. Applied Mechanics Reviews, 2002, 55(5): B90.

[26] GREAVES G N, GREER A, LAKES R S, et al. Poisson's ratio and modern materials. Nature Materials, 2011, 10(11): 823-837.

[27] YANG J, MEISNER G P, CHEN L. Strain field fluctuation effects on lattice thermal conductivity of $\mathrm{ZrNiSn}$-based thermoelectric compounds. Applied Physics Letters, 2004, 85(7): 1140-1142.

[28] MEISNER G P, MORELLI D T, HU S, et al. Structure and lattice thermal conductivity of fractionally filled skutterudites: solid solutions of fully filled and unfilled end members. Physical Review Letters, 1998, 80(16): 3551-3554.

[29] PLIRDPRING T, KUROSAKI K, KOSUGA A, et al. Chalcopyrite $\mathrm{CuGaTe}_{2}$ : a high-efficiency bulk thermoelectric material. Advanced Materials, 2012, 24(27): 3622-3626.

[30] CHENG N, LIU R, BAI S, et al. Enhanced thermoelectric performance in $\mathrm{Cd}$ doped $\mathrm{CuInTe}_{2}$ compounds. Journal of Applied Physics, 2014, 115(16): 163705.

[31] QIN Y, QIU P, LIU R, et al. Optimized thermoelectric properties in pseudocubic diamond-like $\mathrm{CuGaTe}_{2}$ compounds. Journal of Materials Chemistry A, 2016, 4(4): 1277-1289.

[32] GASCOIN F, OTTENSMANN S, STARK D, et al. Zintl phases as thermoelectric materials: tuned transport properties of the compounds $\mathrm{Ca}_{x} \mathrm{Yb}_{1-\chi} \mathrm{Zn}_{2} \mathrm{Sb}_{2}$. Advanced Functional Materials, 2005, 15(11): 1860-1864.

[33] MAO J, KIM H S, SHUAI J, et al. Thermoelectric properties of materials near the band crossing line in $\mathrm{Mg}_{2} \mathrm{Sn}-\mathrm{Mg}_{2} \mathrm{Ge}-\mathrm{Mg}_{2} \mathrm{Si}$ system. Acta Materialia, 2016, 103: 633-642.

[34] LIU W, TAN X, YIN K, et al. Convergence of conduction bands as a means of enhancing thermoelectric performance of n-type $\mathrm{Mg}_{2} \mathrm{Si}_{1-x} \mathrm{Sn}_{x}$ solid solutions. Physical Review Letters, 2012, 108(16): 166601 .

[35] BANERJEE S, RAMAKRISHNAN T V, DASGUPTA C. Phenomenological Ginzburg-Landau-like theory for superconductivity in the cuprates. Physical Review B, 2011, 83(2): 024510.

[36] LIU W, LUKAS K C, MCENANEY K, et al. Studies on the $\mathrm{Bi}_{2} \mathrm{Te}_{3}-\mathrm{Bi}_{2} \mathrm{Se}_{3}-\mathrm{Bi}_{2} \mathrm{~S}_{3}$ system for mid-temperature thermoelectric energy conversion. Energy \& Environmental Science, 2013, 6(2): 552-560.

[37] YAMINI S A, WANG H, GIBBS Z M, et al. Chemical composition tuning in quaternary $\mathrm{p}$-type $\mathrm{Pb}$-chalcogenides - a promising strategy for enhanced thermoelectric performance. Physical Chemistry Chemical Physics, 2014, 16(5): 1835-1840.

[38] KORKOSZ R J, CHASAPIS T C, LO S H, et al. High ZT in p-type $(\mathrm{PbTe})_{1-2 x}(\mathrm{PbSe})_{x}(\mathrm{PbS})_{x}$ thermoelectric materials. Journal of the American Chemical Society, 2014, 136(8): 3225-3237.

[39] HU L, ZHANG Y, WU H, et al. Entropy engineering of SnTe: multi-principal-element alloying leading to ultralow lattice thermal conductivity and state-of-the-art thermoelectric performance. Advanced Energy Materials, 2018, 8(29): 1802116.

[40] ZHAO S Y, CHEN R, LI J Q, et al. Synergistic effects on thermoelectric properties of $\mathrm{Sn}_{0.5} \mathrm{Ge}_{0.4875} \mathrm{Te}$ with $\mathrm{Pb}$ alloying. Journal of Alloys and Compounds, 2019, 777: 1334-1339.

[41] POSFAI M, BUSECK P R. Djurleite, digenite, and chalcocite: intergrowths and transformations. American Mineralogist, 1994, 79(3/4): 308-315.

[42] GULAY L, DASZKIEWICZ M, STROK O, et al. Crystal structure of $\mathrm{Cu}_{2} \mathrm{Se}$. Chemistry of Metals and Alloys, 2011, 4(3/4): 200-205.

[43] PASHINKIN A, FEDOROV V. Phase equilibria in the $\mathrm{Cu}-\mathrm{Te}$ system. Inorganic Materials, 2003, 39(6): 539-554.

[44] HE Y, LU P, SHI X, et al. Ultrahigh thermoelectric performance in mosaic crystals. Advanced Materials, 2015, 27(24): 3639-3644.

[45] ZHAO K, QIU P, SONG Q, et al. Ultrahigh thermoelectric performance in $\mathrm{Cu}_{2-y} \mathrm{Se}_{0.5} \mathrm{~S}_{0.5}$ liquid-like materials. Materials Today Physics, 2017, 1: 14-23.

[46] ZHAO K, ZHU C, QIU P, et al. High thermoelectric performance and low thermal conductivity in $\mathrm{Cu}_{2-y} \mathrm{~S}_{1 / 3} \mathrm{Se}_{1 / 3} \mathrm{Te}_{1 / 3}$ liquid-like materials with nanoscale mosaic structures. Nano Energy, 2017, 42: 43-50.

[47] WELDERT K S, ZEIER W G, DAY T W, et al. Thermoelectric transport in $\mathrm{Cu}_{7} \mathrm{PSe}_{6}$ with high copper ionic mobility. Journal of the American Chemical Society, 2014, 136(34): 12035-12040.

[48] CHEN R, QIU P, JIANG B, et al. Significantly optimized thermoelectric properties in high-symmetry cubic $\mathrm{Cu}_{7} \mathrm{PSe}_{6}$ compounds via entropy engineering. Journal of Materials Chemistry A, 2018, 6(15): 6493-6502.

[49] JIANG B, QIU P, CHEN H, et al. Entropy optimized phase transitions and improved thermoelectric performance in n-type liquid-like $\mathrm{Ag}_{9} \mathrm{GaSe}_{6}$ materials. Materials Today Physics, 2018, 5: 20-28.

[50] JIANG B, QIU P, CHEN H, et al. An argyrodite-type $\mathrm{Ag}_{9} \mathrm{GaSe}_{6}$ liquid-like material with ultralow thermal conductivity and high thermoelectric performance. Chemical Communications, 2017, 53(85): 11658-11661.

[51] PEI Y, LALONDE A, IWANAGA S, et al. High thermoelectric figure of merit in heavy hole dominated PbTe. Energy \& Environmental Science, 2011, 4(6): 2085-2089.

[52] LI J, ZHANG X, CHEN Z, et al. Low-symmetry rhombohedral GeTe thermoelectrics. Joule, 2018, 2(5): 976-987.

[53] ZHAO L D, LO S H, ZHANG Y, et al. Ultralow thermal conductivity and high thermoelectric figure of merit in SnSe crystals. Nature, 2014, 508(7496): 373-377.

[54] FAN Z, WANG H, WU Y, et al. Thermoelectric performance of PbSnTeSe high-entropy alloys. Materials Research Letters, 2017, 5(3): 187-194.

[55] WU Y, NAN P, CHEN Z, et al. Manipulation of band degeneracy and lattice strain for extraordinary PbTe thermoelectrics. Research, 2020, 2020: 8151059 .

[56] RAOUX S, MUñOZ B, CHENG H Y, et al. Phase transitions in Ge-Te phase change materials studied by time-resolved X-ray diffraction. Applied Physics Letters, 2009, 95(14): 143118.

[57] ALPTEKIN S. Structural phase transition of SnSe under uniaxial stress and hydrostatic pressure: an $a b$ initio study. Journal of Molecular Modeling, 2011, 17(11): 2989-2994.

[58] MUIR J A, BEATO V. Phase transformations in the system GeSeGeTe. Journal of the Less Common Metals, 1973, 33(3): 333-340.

[59] WIEDEMEIER H, SIEMERS P. The thermal expansion and high temperature transformation of GeSe. Zeitschrift für Anorganische und Allgemeine Chemie, 1975, 411(1): 90-96.

[60] SIST M, GATTI C, NØRBY P, et al. High-temperature crystal structure and chemical bonding in thermoelectric germanium selenide (GeSe). Chemistry-A European Journal, 2017, 23(28): 6888-6895.

[61] HUANG Z, MILleR S A, GE B, et al. High thermoelectric performance of new rhombohedral phase of GeSe stabilized through alloying with $\mathrm{AgSbSe}_{2}$. Angewandte Chemie International Edition, 2017, 129(45): 14301-14306.

[62] QIU Y, JIN Y, WANG D, et al. Realizing high thermoelectric performance in GeTe through decreasing the phase transition temperature via entropy engineering. Journal of Materials Chemistry A, 2019, 7(46): 26393-26401.

[63] ZHANG R Z, GUCCI F, ZHU H, et al. Data-driven design of ecofriendly thermoelectric high-entropy sulfides. Inorganic Chemistry, 2018, 57(20): 13027-13033.

[64] FAN Z, WANG H, WU Y, et al. Thermoelectric high-entropy alloys with low lattice thermal conductivity. RSC Advances, 2016, 6(57): 52164-52170.

[65] RAPHEL A, VIVEKANANDHAN P, KUMARAN S. High entropy phenomena induced low thermal conductivity in $\mathrm{BiSbTe}_{1.5} \mathrm{Se}_{1.5}$ thermoelectric alloy through mechanical alloying and spark plasma sintering. Materials Letters, 2020, 269: 127672.

[66] YAN J, LIU F, MA G, et al. Suppression of the lattice thermal conductivity in NbFeSb-based half-Heusler thermoelectric materials through high entropy effects. Scripta Materialia, 2018, 157: 129-134.

[67] SAKURADA S, SHUTOH N. Effect of Ti substitution on the thermoelectric properties of $(\mathrm{Zr}, \mathrm{Hf}) \mathrm{NiSn}$ half-Heusler compounds. Applied Physics Letters, 2005, 86(8): 082105.

[68] VOLYKHOV A, YASHINA L, TAMM M, et al. Phase equilibria in ternary reciprocal systems based on IV-VI compounds. Inorganic Materials 2009, 45(9): 968-974.

[69] WANG Y Y, ROGADO N S, CAVA R J, et al. Spin entropy as the likely source of enhanced thermopower in $\mathrm{Na}_{x} \mathrm{Co}_{2} \mathrm{O}_{4}$. Nature, 2003, 423(6938): 425-428.

[70] EMIN D. Enhanced Seebeck coefficient from carrier-induced vibrational softening. Physical Review B, 1999, 59(9): 6205-6210.

[71] HAN C G, QIAN X, LI Q K, et al. Giant thermopower of ionic gelatin near room temperature. Science, 2020, 368(6495): 1091-1098. 